OPEN ACCESS

Edited by: Jan Kassubek,

University of UIm, Germany

Reviewed by:

Cristian E. Leyton,

The University of Sydney, Australia

Volker Rasche,

University of UIm, Germany

*Correspondence:

Byung Se Choi

byungse.choi@gmail.com

Specialty section:

This article was submitted to Applied Neuroimaging,

a section of the journal

Frontiers in Neurology

Received: 24 July 2020 Accepted: 09 March 2021

Published: 07 April 2021

Citation:

Cho SJ, Choi BS, Bae YJ, Baik SH, Sunwoo L and Kim JH (2021) Image Findings of Acute to Subacute Craniocervical Arterial Dissection on Magnetic Resonance Vessel Wall Imaging: A Systematic Review and Proportion Meta-Analysis. Front. Neurol. 12:586735 doi: 10.3389/fneur.2021.586735

\section{Image Findings of Acute to Subacute Craniocervical Arterial Dissection on Magnetic Resonance Vessel Wall Imaging: A Systematic Review and Proportion Meta-Analysis}

\author{
Se Jin Cho, Byung Se Choi*, Yun Jung Bae, Sung Hyun Baik, Leonard Sunwoo and \\ Jae Hyoung Kim
}

Department of Radiology, Seoul National University College of Medicine, Seoul National University Bundang Hospital, Seongnam, South Korea

Background and Purpose: This systematic review and meta-analysis aimed to evaluate the pooled proportion of image findings of acute to subacute craniocervical arterial dissection (AD) direct signs on magnetic resonance vessel wall imaging (MR-VWI) and to identify factors responsible for the heterogeneity across the included studies.

Methods: A systematic literature search in the Ovid-MEDLINE and EMBASE databases was performed for studies published on the relevant topic before April 14, 2020. Pooled sensitivity and specificity values and their 95\% confidence intervals (Cls) were calculated using bivariate random-effects modeling. Meta-regression analyses were also performed to determine factors influencing heterogeneity.

Results: Eleven articles with data for 209 patients with acute to subacute craniocervical AD who underwent MR-VWI were included in this systematic review and meta-analysis. The most common findings on MR-WWI were wall hematoma (84\%; 95\% Cl, 71\%-92\%), abnormal enhancement (72\%; 95\% Cl, 49\%-88\%), aneurysmal dilatation (71\%, 95\% $\mathrm{Cl}, 53 \%-84 \%$ ), and intimal flap or double lumen signs (49\%; 95\% $\mathrm{Cl}, 29 \%-71 \%)$. Among the potential covariates of heterogeneity, the presence of contrast-enhanced T1-weighted imaging (CE-T1WI) within the MR-VWI sequence combination significantly affected the pooled proportion of the intimal flap or double lumen signs.

Conclusion: Wall hematoma and intimal flap or double lumen signs were the most common and least common direct sign image findings, respectively, on MR-VWI in patients with acute to subacute craniocervical AD. Furthermore, the absence of CE-T1WI in MR-VWI protocol was the cause of heterogeneity for the detection of the intimal flap or double lumen signs. This data may help improve MR-WWI interpretation and enhance the understanding of the radiologic diagnosis of craniocervical AD.

Keywords: arteries, dissection, magnetic resonance imaging, systematic review, meta-analysis 


\section{INTRODUCTION}

Conventionally, digital subtraction angiography (DSA) had been the method of choice for diagnosing craniocervical arterial dissection (AD) using findings such as double lumen and pearl and string signs $(1,2)$. Due to the invasiveness of DSA, magnetic resonance angiography (MRA) emerged as a non-invasive alternative to the luminal evaluation of craniocervical AD (3). Conventional MRA can provide information about geometric vascular changes, hemodynamic alterations, and collateral blood vessel development, but these are indirect signs, and there are inevitable limitations for the depiction and evaluation of arterial wall pathology $(1,2)$. Owing to its non-invasiveness and superior performance compared to luminal angiography, high-resolution magnetic resonance vessel wall imaging (MR-VWI) is now widely used for the diagnosis of $\operatorname{AD}(2,4-6)$.

However, despite improving the diagnosis of AD considerably, MR-VWI remains a subject of active ongoing research in terms of aspects such as sequence combinations, spatial resolution, and acquisition techniques (2, 4-6). Correspondingly, studies reporting image findings of direct signs of vascular wall pathology have focused on positive findings on MR-VWI in patients with acute or subacute craniocervical $\mathrm{AD}(4,7-15)$. With this in mind, the recognition of direct signs of MR-VWI would enhance a comprehensive understanding of the radiologic diagnosis of craniocervical AD. Furthermore, the factors responsible for the heterogeneity in techniques, protocols, and image findings of direct signs across studies need to be identified and crossvalidated. In this context, this systematic review and metaanalysis aimed to evaluate the pooled proportion of image findings of direct signs of craniocervical AD on MR-VWI and to identify factors responsible for heterogeneity across the included studies. To the best of our knowledge, this is the first systematic review and meta-analysis that pools image findings on MR-VWI in patients with $\mathrm{AD}$.

\section{MATERIALS AND METHODS}

This study was performed according to the Preferred Reporting Items for Systematic Reviews and Meta-Analyses (PRISMA) guidelines (16).

\section{Literature Search}

A search of the MEDLINE and EMBASE databases was performed to find original literature reporting image findings of direct signs on MR-VWI for patients with acute to subacute craniocervical AD. The following search terms were used: [(Magnetic resonance vessel wall imaging) OR ( $\mathrm{MR}^{*}$ vessel wall imaging) OR (vessel wall imaging) OR (vessel wall $\mathrm{MR}^{*}$ ) OR (high-resolution MR*) OR (high-resolution magnetic resonance)] AND [(dissect*)]. No initial search date was set, and the literature search was updated until April 14, 2020. The search

Abbreviations: $\mathrm{AD}$, arterial dissection; $\mathrm{BB}$, black blood; CE-T1WI, contrast enhanced T1-weighted imaging; MRA, magnetic resonance angiography; MRVWI, magnetic resonance vessel wall imaging. was limited to publications in English. The bibliographies of relevant articles were searched to identify other relevant articles.

\section{Inclusion Criteria}

Studies satisfying the following criteria were included: (1) involved patients with acute to subacute craniocervical AD; (2) used MR-VWI as the index test; and (4) contained sufficient information regarding the proportion of image findings of direct signs in acute to subacute craniocervical AD on MR-VWI.

\section{Exclusion Criteria}

The following articles or article subset types were excluded: (1) case reports or series including fewer than five patients; (2) letters, editorials, conference abstracts, systematic reviews or meta-analyses, consensus statements, guidelines, and review articles; (4) articles not focusing on the current topic; (5) articles with, or with suspicion of, overlapping populations; and (6) articles containing insufficient information on the proportion of image findings of direct signs of acute to subacute craniocervical AD on MR-VWI.

Two radiologists, S.J.C and B.S.C, with 7 and 19 years of experience in neuroimaging, respectively, independently performed the literature search and selection.

\section{Data Extraction}

The following data were extracted using standardized forms according to the PRISMA guidelines (16): (1) characteristics and demographic data of the included studies: author names, year of publication, institution, country of origin, period of patient recruitment, study design (prospective vs. retrospective), patient number, male-to-female ratio, mean age of enrolled patients (adult or not), number of patients with acute to subacute craniocervical $\mathrm{AD}$, reference standard, clinical diagnostic criteria for $\mathrm{AD}$, mean interval days from symptom to $\mathrm{MR}-\mathrm{VWI}$ and their ranges, $\mathrm{AD}$ location (intracranial vs. extracranial), and mechanism of the $\mathrm{AD}$ (spontaneous vs. traumatic); (2) patient symptoms and underlying risk factors at initial presentation; (4) MR-VWI characteristics: MRI machine and vendor, magnetic field strength, MRI dimension (2D or 3D), coil type, under-sampling techniques, use of the black blood (BB) technique during image acquisition, MR-VWI sequence combination, repetition time/echo time, field of view, slice thickness, acquisition matrix of T1-weighted imaging (T1WI) as a representative sequence, analytical methods to evaluate MRVWI (subjective or objective), and the interobserver agreement for diagnosis; and (5) the proportion of image findings of direct signs on MR-VWI, luminal stenosis on angiography, and presence of infarction.

$\mathrm{AD}$ was defined as acute stage if it was detected within 3 days of symptom onset, subacute stage 4-60 days, and chronic stage after 60 days (17).

\section{Quality Assessment}

The methodological quality of the included studies was evaluated using tailored questionnaires and the Quality Assessment of Diagnostic Accuracy Studies-2 (QUADAS-2) criteria (18). Two 


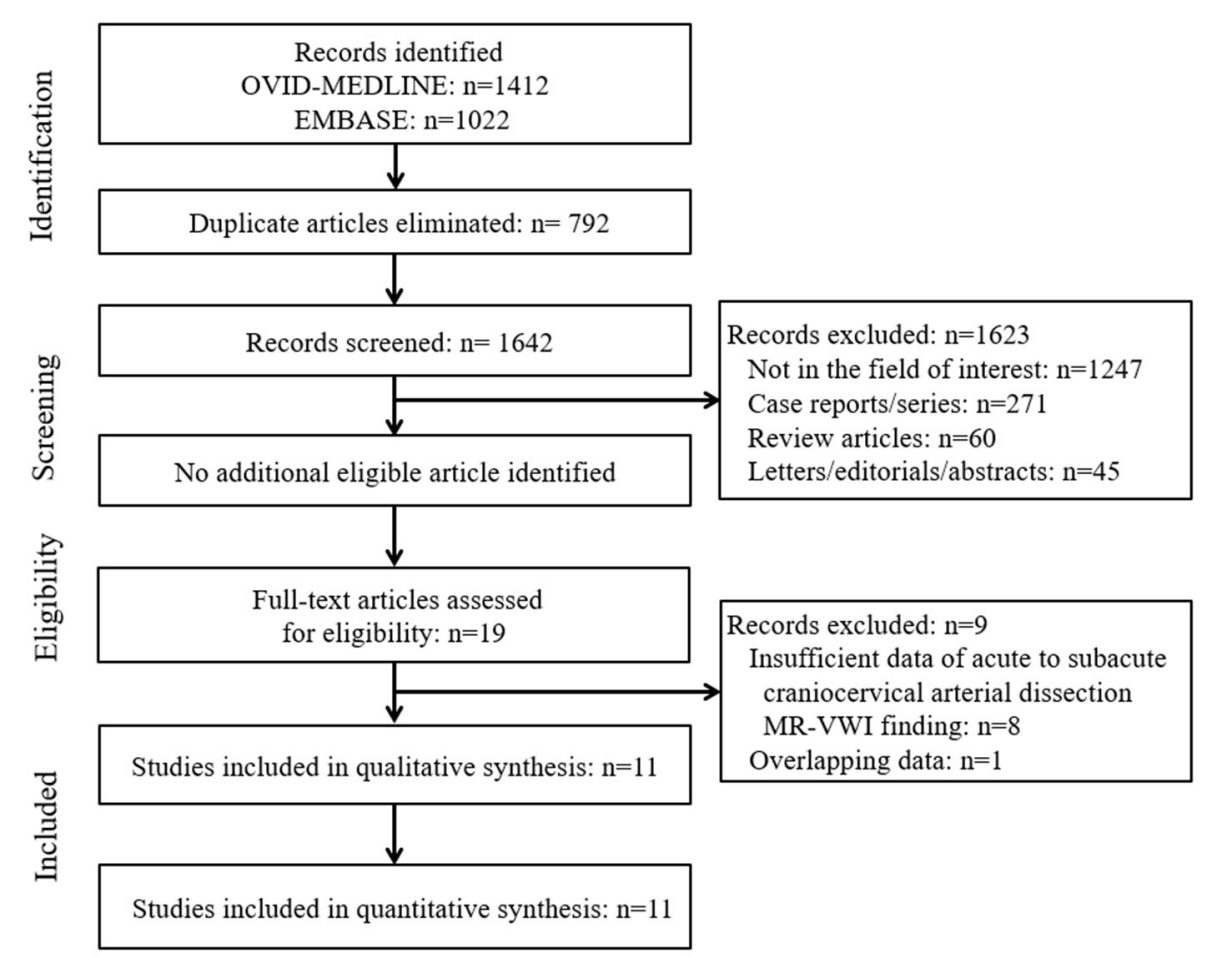

FIGURE 1 | Flow diagram of the study selection process.

reviewers (S.J.C and B.S.C) independently performed data extraction and quality assessment.

\section{Data Synthesis and Analyses}

The primary aim was to describe the demographic data and perform pooled proportion analysis of image findings of direct signs of acute to subacute craniocervical AD on MR-VWI. The secondary aim was to identify factors responsible for the heterogeneity across the included studies by employing meta-regression analysis. Pooled proportions were calculated using an inverse-variance weighting model (19-21). A randomeffects meta-analysis of proportions was utilized to calculate the overall proportions. Study heterogeneity was evaluated using the Higgins inconsistency index $\left(I^{2}\right)$, with substantial heterogeneity indicated by an $I^{2}$ value $>50 \%$ (22). In addition, meta-regression analysis was performed for the pooled proportion of image findings to determine the factor for the heterogeneity. All statistical analyses were conducted by one author (S.J.C, with 3 years of experience in conducting systematic reviews and metaanalysis) using the "meta" package of $\mathrm{R}$ (version 3.6.3; http:// www.r-project.org/).

\section{RESULTS}

\section{Literature Search}

The systematic literature search (Figure 1) initially identified 2,434 articles. After removing 792 duplicates, screening of the remaining 1,642 titles and abstracts yielded 19 potentially eligible articles. No additional articles were identified after searching their bibliographies. After full-text reviews of these 19 articles, eight were excluded because they contained insufficient information in terms of the proportion of image findings of acute to subacute craniocervical AD on MR-VWI (2, 5, $6,23-27)$ and one because of suspected population overlap (28). Finally, 11 articles were included in the final analysis $(4,7-15,29)$.

\section{Characteristics of the Included Studies}

The total number of patients with AD across all studies was 327 (range, 6 to 118 patients). The number of dissected arteries across all studies was 372 (range, 6 to 145) (Table 1). The mean patient age across studies ranged from 40.4 to 55 years. Except for one patient in one study, all studies included adults (10). Eight of the included articles were retrospective in design $(4,7,8,11$, $13-15,29)$, whereas three studies were prospective $(9,10,12)$. The reference standard for diagnosing $\mathrm{AD}$ was a combination of clinical, radiological, and luminal angiography diagnoses in three studies $(12,14,29)$ and of clinical, radiological, luminal angiography, and follow-up MRI in eight studies $(4,7-11,13,15)$. Two of these studies specifically used the spontaneous AD criteria for clinical diagnosis $(8,15)$, and two used the Strategies Against Stroke Study for Young Adults in Japan criteria $(11,14)$. The mean intervals from symptom to MR-VWI were shorter than 10 days in six of the included studies $(4,9,12-15)$ and equal to or longer than 10 days in four $(7,8,11,29)$; the relevant information was not specified in a remaining article (10). AD 
TABLE 1 | Characteristics of the included studies.

\begin{tabular}{|c|c|c|c|c|c|c|c|c|c|c|c|c|c|}
\hline Source & Affiliation & $\begin{array}{l}\text { Period of patient } \\
\text { recruitment }\end{array}$ & $\begin{array}{l}\text { Study } \\
\text { design }\end{array}$ & Pt. no. & AD no. & $\begin{array}{c}\text { Mean } \\
\text { age }\end{array}$ & $\begin{array}{l}\text { Male/ } \\
\text { female }\end{array}$ & Adult & $\begin{array}{l}\text { Reference } \\
\text { standard/criteria* }\end{array}$ & $\begin{array}{l}\text { Mean days } \\
\text { from Sx. to } \\
\text { VWI (range) }\end{array}$ & $\begin{array}{l}\text { Location of } \\
\text { AD }\end{array}$ & $\begin{array}{l}\text { Intracranial vs. } \\
\text { extracranial }\end{array}$ & $\begin{array}{l}\text { Mechanism of } \\
A D\end{array}$ \\
\hline $\begin{array}{l}\text { Coppenrath } \\
\text { et al. (12) }\end{array}$ & $\begin{array}{l}\text { Ludwig Maximilian } \\
\text { University Munich, } \\
\text { Germany }\end{array}$ & $\begin{array}{l}\text { August 2007-June } \\
2010\end{array}$ & Pros. & 33 & 44 & 47 & $19 / 14$ & All & $\begin{array}{l}\text { Clinico. + Radio. + } \\
\text { Angio./NA }\end{array}$ & $N A(<7)$ & $\begin{array}{l}29 \text { ICA, } 15 \\
\text { VBA }\end{array}$ & NA & All spontaneous \\
\hline $\begin{array}{l}\text { Cuvinciuc et al. } \\
\text { (7) }\end{array}$ & $\begin{array}{l}\text { University Hospitals of } \\
\text { Geneva, Switzerland }\end{array}$ & NA & Retro. & 14 & 14 & 45.1 & $11 / 3$ & All & $\begin{array}{l}\text { Clinico. + Radio. + Angio. } \\
\text { + FU Radio./NA }\end{array}$ & $12(1-45)$ & 9 ICA, 5 VBA & Both & $\begin{array}{l}10 \text { spontaneous, } 4 \\
\text { traumatic }\end{array}$ \\
\hline Han et al. (4) & $\begin{array}{l}\text { Ajou University Medical } \\
\text { Center, Republic of } \\
\text { Korea }\end{array}$ & $\begin{array}{l}\text { March } \\
2012-\text { October } \\
2013\end{array}$ & Retro. & 33 & 35 & 51.1 & $28 / 3$ & All & $\begin{array}{l}\text { Clinico. + Radio. + Angio. } \\
\text { + FU Radio./NA }\end{array}$ & $2.5(1-7)$ & $\begin{array}{l}1 \mathrm{BA}, 9 \mathrm{PICA}, \\
25 \mathrm{VA}\end{array}$ & Intracranial & NA \\
\hline Jung et al. (11) & $\begin{array}{l}\text { Asan Medical Center, } \\
\text { Republic of Korea }\end{array}$ & $\begin{array}{l}\text { April } \\
\text { 2012-February } \\
2015\end{array}$ & Retro. & 26 & 28 & 47 & $17: 9$ & All & $\begin{array}{l}\text { Clinico. + Radio. + Angio. } \\
\text { + FU Radio./SASSY-Japan }\end{array}$ & $10(1-30)$ & $\begin{array}{l}6 \text { ACA, } 4 \\
\text { MCA; } 18 \text { VBA }\end{array}$ & Intracranial & All spontaneous \\
\hline Kano et al. (15) & $\begin{array}{l}\text { Tosei General Hospital, } \\
\text { Japan }\end{array}$ & $\begin{array}{l}\text { March 2016-July } \\
2019\end{array}$ & Retro. & 6 & 6 & 44.8 & $4: 2$ & All & $\begin{array}{l}\text { Clinico. + Radio. + Angio. } \\
\text { + FU Radio./SCADS }\end{array}$ & $5(2-14)$ & All PICA & Intracranial & All spontaneous \\
\hline Li et al. (10) & $\begin{array}{l}\text { Beijing Tiantan } \\
\text { Hospital, China }\end{array}$ & $\begin{array}{l}\text { June } \\
2012-S e p t e m b e r \\
2014\end{array}$ & Pros. & 24 & 24 & 45 & $21: 3$ & Almostł & $\begin{array}{l}\text { Clinico. + Radio. + Angio. } \\
\text { + FU Radio./NA }\end{array}$ & $N A(<14)$ & $\begin{array}{l}11 \text { ICA, } 3 \\
\text { MCA, } 10 \text { VBA }\end{array}$ & Both & All spontaneous \\
\hline Natori et al. (9) & $\begin{array}{l}\text { Iwate Medical } \\
\text { University, Japan }\end{array}$ & $\begin{array}{l}\text { April 2011-March } \\
2013\end{array}$ & Pros. & 16 & 16 & 55 & NA & All & $\begin{array}{l}\text { Clinico. + Radio. + Angio. } \\
\text { + FU Radio./NA }\end{array}$ & $9.5(0-37)$ & All VBA & Intracranial & All spontaneous \\
\hline $\begin{array}{l}\text { Ogawa et al. } \\
\text { (13) }\end{array}$ & $\begin{array}{l}\text { Nagoya City University } \\
\text { Graduate School of } \\
\text { Medical Sciences, } \\
\text { Japan }\end{array}$ & $\begin{array}{l}\text { January } \\
2015-\text { February } \\
2017\end{array}$ & Retro. & 12 & 14 & 52 & 9: 3 & All & $\begin{array}{l}\text { Clinico. + Radio. + Angio. } \\
\text { + FU Radio./NA }\end{array}$ & $7.3(1-21)$ & All VBA & Intracranial & NA \\
\hline Sakurai et al. (8) & $\begin{array}{l}\text { Nagoya City University } \\
\text { Graduate School of } \\
\text { Medical Sciences, } \\
\text { Japan }\end{array}$ & $\begin{array}{l}\text { November } \\
\text { 2009-April } 2011\end{array}$ & Retro. & $15 \dagger$ & $17 \dagger$ & $51 \dagger$ & NA† & All & $\begin{array}{l}\text { Clinico. + Radio. + Angio. } \\
\text { + FU Radio./SCADS }\end{array}$ & $22(2-57)$ & All VBA & Intracranial & All spontaneous \\
\hline Wu et al. (29) & $\begin{array}{l}\text { Xuanwu Hospital, } \\
\text { China }\end{array}$ & $\begin{array}{l}\text { September } \\
\text { 2013-September } \\
2018\end{array}$ & Retro. & 145 & 145 & 40.4 & 95,50 & All & $\begin{array}{l}\text { Clinico. + Radio. + } \\
\text { Angio./NA }\end{array}$ & $12(6-20)$ & $\begin{array}{l}77 \text { VBA, } 66 \\
\text { ICA, } 1 \text { PCA, } \\
21 \text { multiple }\end{array}$ & Both & NA \\
\hline Yun et al. (14) & $\begin{array}{l}\text { Busan Paik Hospital, } \\
\text { Republic of Korea }\end{array}$ & $\begin{array}{l}\text { May 2013-August } \\
2017\end{array}$ & Retro. & 27 & 29 & 52.5 & 15,12 & All & $\begin{array}{l}\text { Clinico. + Radio. + } \\
\text { Angio./SASSY-Japan }\end{array}$ & $4(0-60)$ & All VBA & Intracranial & All spontaneous \\
\hline
\end{tabular}

ACA, anterior cerebral artery; BA, basilar artery; AD, arterial dissection; MCA, middle cerebral artery; ICA, internal cerebral artery; NA, not available; no., number; Pt., patient, PICA, posterior inferior cerebellar artery; Pros, prospective; Retro, retrospective; SX., symptom; VA, vertebral artery; VBA, vertebrobasilar artery; VWI, vessel wall imaging; SCADS, Spontaneous coronary artery dissection criteria; SASSY-Japan. Strategies Against Stroke Study for Young Adults

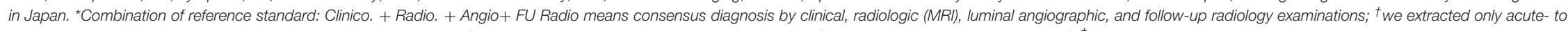
subacute-staged AD of 15 patients' data from 20 AD of 18 patients (the mean age was that of 18 patients, and the male/female ratio of 18 patients were 14:4); ₹except only a 1-year-old female. 
TABLE 2 | Pooled proportion of symptoms at presentation and the risk factors of patients with arterial dissection.

\begin{tabular}{|c|c|c|c|c|c|c|c|c|c|c|c|c|}
\hline Source & \multicolumn{7}{|c|}{ Symptom } & \multicolumn{5}{|c|}{ Risk factor } \\
\hline Coppenrath et al. (12) & 10 & 17 & 0 & 4 & 4 & 0 & 3 & 12 & 18 & 10 & 0 & 5 \\
\hline Cuvinciuc et al. (7) & 3 & 7 & 1 & 1 & 7 & 1 & 2 & NA & NA & NA & NA & NA \\
\hline Han et al. (4) & 16 & 5 & 20 & 6 & 0 & 0 & 12 & 14 & 17 & 5 & 6 & 2 \\
\hline Li et al. (10) & 7 & NA & NA & NA & NA & NA & NA & 13 & 10 & 0 & 6 & 0 \\
\hline Natori et al. (9) & NA & NA & NA & NA & NA & NA & NA & 4 & 0 & 1 & 2 & 0 \\
\hline Ogawa et al. (13) & 8 & 5 & 3 & 0 & 0 & 0 & 2 & NA & NA & NA & NA & NA \\
\hline Sakurai et al. (8) & 15 & 5 & 1 & 0 & 0 & 1 & 0 & 4 & 3 & 5 & 0 & 1 \\
\hline Wu et al. (29) & NA & NA & NA & NA & NA & NA & NA & 17 & 33 & 27 & NA & 57 \\
\hline
\end{tabular}

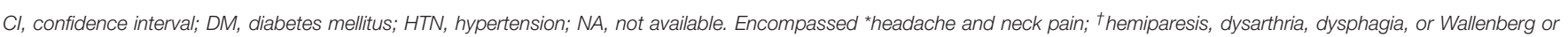
lateral medullary syndrome; ${ }^{\ddagger}$ vomiting, nausea, etc.; and ${ }^{\S}$ familial history of risk factor, coronary artery disease, etc.

was located exclusively in posterior circulation in six studies $(4,8,9,13-15)$ and predominantly in posterior circulation (in more than half the cases) in two studies $(11,29)$; the locations were variable in the other studies $(7,10,12)$. Seven studies only evaluated intracranial arteries $(4,8,9,11,13-15)$ and three studies evaluated both intracranial and extracranial arteries (7, 10, 29); the relevant information was not specified in a remaining article (12).

All but three of the studies included spontaneous AD (8$12,14,15)$; one study partly included traumatic $\mathrm{AD}(7)$, and three studies had unclear information in this regard $(4,13,29)$. The pooled proportion of symptoms at presentation and the underlying risk factors of patients are presented in Table 2. The most common symptoms were pain [44\%; confidence interval (CI), 32\%-57\%], motor weakness (33\%; 95\% CI, $22 \%-46 \%)$, and dizziness (21\%; 95\% CI, 8\%-45\%). Common underlying risk factors were smoking (31\%; 95\% CI, 22\%-43\%), hypertension (29\%; 95\% CI, 19\%-43\%), and dyslipidemia (20\%; 95\% CI, 14\%-26\%).

\section{MR-VWI-Associated Characteristics of Included Studies}

In seven studies, 3-T scanners were used $(4,10-14,29)$ and $1.5-\mathrm{T}$ scanner in four (Table 3) $(7-9,15)$. Eight studies used 3D-MRI $(7-11,13,15,29)$, one used 2D-MRI (12), and one used either 2D- or 3D-MRI (14); the technique used was not specified in one study (4). The included studies used different coil types. Three studies acquired MRI data using under-sampling techniques, all of which were parallel imaging techniques $(8,9,15)$. Five studies used the BB technique $(4,8,10,12,13)$. Regarding MR-VWI sequence combinations, all studies included T1WIbased sequences, whereas the use of contrast enhanced-T1WI (CE-T1WI), T2WI, and proton density imaging was variable. T1WI (as a representative MR-VWI sequence) acquisition parameter details are presented in Table 3. Slice thicknesses $<1 \mathrm{~mm}$ were used in seven studies $(7-10,13,15,29)$; in two of these, the thickness was $<0.6 \mathrm{~mm}(7,9)$. Nine studies used subjective image analysis $(4,7-10,12,13,15,29)$, whereas two studies added objective measurement for the image findings $(11,14)$. Four studies revealed the interobserver agreement in diagnosing $\mathrm{AD}$ between two observers, which were all moderate to excellent (kappa range, 0.73-1) $(4,7,9,13)$.

\section{Pooled Proportions of Image Findings on MR-VWI}

The pooled proportions of the presence of wall hematoma, abnormal enhancement, aneurysmal dilatation, and intimal flap or double lumen signs on MR-VWI for patients with craniocervical $\mathrm{AD}$ were $86 \%$ (95\% CI, 74\%-93\%), 75\% (95\% CI, 56\%-87\%), 71\% (95\% CI, 53\%-84\%), and $47 \%$ (95\% CI, 30\%-65\%), respectively (Table 4, Figure 2). Heterogeneity regarding image findings was noted across studies $\left(I^{2}=\right.$ $72 \%-84 \%)$. Pooled proportions of luminal stenosis or occlusion on luminal angiography and the presence of infarction were $76 \%$ (95\% CI, 55\%-89\%) and 55\% (95\% CI, 39\%-70\%), respectively. Heterogeneity regarding luminal stenosis or occlusion and the presence of infarction across studies were noted $\left(I^{2}=77 \%\right.$ and $81 \%$, respectively).

\section{Meta-Regression}

We used meta-regression analysis to determine the causes of heterogeneity. The covariates evaluated were mean interval from symptom to MR-VWI ( $<10$ vs. $\geq 10$ days), a predominance of posterior circulation in terms of $\mathrm{AD}$ location (more than half), magnetic resonance field strength $(1.5 \mathrm{~T}$ vs. $3 \mathrm{~T})$, performance via the $\mathrm{BB}$ technique during image acquisition, presence of $\mathrm{CE}$ T1WI within the MR-VWI sequence combination, repetition 
TABLE 3 | Characteristics of magnetic resonance vessel wall imaging.

\begin{tabular}{|c|c|c|c|c|c|c|c|c|c|c|c|c|c|c|}
\hline \multirow[t]{2}{*}{ Source } & \multicolumn{7}{|c|}{ Protocol } & \multirow{2}{*}{$\frac{\mathrm{T} 1}{\mathrm{TR} / \mathrm{TE},}$} & \multirow{2}{*}{$\frac{\mathrm{T} 1 \mathrm{WI}}{\mathrm{FOV}, \mathrm{mm}^{2} \dagger}$} & \multirow{2}{*}{$\begin{array}{c}\text { T1WI } \\
\begin{array}{c}\text { Slice } \\
\text { thickness, } \\
\text { mm }\end{array}\end{array}$} & \multirow{2}{*}{$\frac{\text { T1WI }}{\begin{array}{c}\text { Acquisition } \\
\text { matrix } \dagger\end{array}}$} & \multirow{2}{*}{$\begin{array}{l}\text { Assessment } \\
\text { Methods } \\
\text { (reader no.) }\end{array}$} & \multirow[b]{2}{*}{$\begin{array}{l}\text { Interobserver } \\
\text { agreement } \\
\text { for diagnosis }\end{array}$} & \multirow[b]{2}{*}{$\begin{array}{l}\text { Visual (subj.) } \\
\text { assessment }\end{array}$} \\
\hline & Machine & $\mathbf{T}$ & D & Coil & $\begin{array}{l}\text { Under- } \\
\text { sampling }\end{array}$ & BB & $\begin{array}{l}\text { Sequence } \\
\text { combination }\end{array}$ & & & & & & & \\
\hline $\begin{array}{l}\text { Coppenrath } \\
\text { et al. (12) }\end{array}$ & $\begin{array}{l}\text { Verio, } \\
\text { Siemens }\end{array}$ & 3 & 2 & $\begin{array}{l}\text { Flexible } \\
\text { 4-channel } \\
\text { carotid } \\
\text { surface coil }\end{array}$ & NA & Yes & $\begin{array}{l}\text { T1WI, CE-T1WI, } \\
\text { CE-MRA }\end{array}$ & $800 / 12$ & $160 \times 120$ & 2 & $240 \times 320$ & Subj. (2) & NA & $\begin{array}{l}\text { Vessel wall } \\
\text { hematoma, abnormal } \\
\text { enhancement, luminal } \\
\text { occlusion, and } \\
\text { presence of infarction }\end{array}$ \\
\hline $\begin{array}{l}\text { Cuvinciuc et al. } \\
\text { (7) }\end{array}$ & $\begin{array}{l}\text { Espree MRI } \\
\text { scanner, } \\
\text { Siemens }\end{array}$ & 1.5 & 3 & $\begin{array}{l}\text { Standard } \\
\text { head and } \\
\text { neck coil }\end{array}$ & NA & No & $\begin{array}{l}\text { T1WI (SPACE), } \\
\text { CE-MRA, }\end{array}$ & $750 / 2$ & $230 \times 230$ & 0.45 & $256 \times 256$ & Subj. (2) & Kappa 0.82-1 & Vessel wall hematoma \\
\hline Han et al. (4) & $\begin{array}{l}\text { Intera } \\
\text { Achieva, } \\
\text { Philips }\end{array}$ & 3 & NA & $\begin{array}{l}\text { 16-channel } \\
\text { neurovascular } \\
\text { head coil }\end{array}$ & NA & Yes & $\begin{array}{l}\text { T1WI, T2WI, PD, } \\
\text { CE-T1WI, TOF-MRA }\end{array}$ & $1,000 / 7.9$ & $100 \times 100$ & 2 & $20 \times 200$ & Subj. (2) & Kappa $0.83^{*}$ & $\begin{array}{l}\text { Vessel wall hematoma, } \\
\text { intimal flap or double } \\
\text { lumen, luminal } \\
\text { occlusion, aneurysmal } \\
\text { dilatation, and } \\
\text { presence of infarction }\end{array}$ \\
\hline Jung et al. (11) & $\begin{array}{l}\text { Achieva, } \\
\text { Philips }\end{array}$ & 3 & 3 & $\begin{array}{l}\text { 8-channel } \\
\text { head coil }\end{array}$ & NA & No & $\begin{array}{l}\text { T1WI, T2WI, PD, } \\
\text { CE-T1WI, TOF-MRA }\end{array}$ & $695 / 80$ & $100 \times 100$ & 1 & $512 \times 512$ & $\begin{array}{l}\text { Subj. + Obj. } \\
\text { (2) }\end{array}$ & NA & $\begin{array}{l}\text { Vessel wall hematoma, } \\
\text { intimal flap or double } \\
\text { lumen, abnormal } \\
\text { enhancement, an } \\
\text { extension to other } \\
\text { arteries, and } \\
\text { aneurysmal dilatation }\end{array}$ \\
\hline Kano et al. (15) & $\begin{array}{l}\text { MAGNETOM } \\
\text { Aera, } \\
\text { Siemens }\end{array}$ & 1.5 & 3 & $\begin{array}{l}\text { 20-channel } \\
\text { head and } \\
\text { neck coil }\end{array}$ & $\begin{array}{l}\text { GRAPPA } \\
\text { factor, } 2\end{array}$ & No & $\begin{array}{l}\text { T1WI, T2WI, } \\
\text { TOF-MRA, BPAS }\end{array}$ & $600 / 78$ & $210 \times 210$ & 0.8 & $256 \times 230$ & Subj. (2) & NA & $\begin{array}{l}\text { Vessel wall hematoma, } \\
\text { aneurysmal dilatation, } \\
\text { and luminal stenosis } \\
\text { or occlusion }\end{array}$ \\
\hline Li et al. (10) & $\begin{array}{l}\text { Achieva TX, } \\
\text { Philips }\end{array}$ & 3 & 3 & $\begin{array}{l}\text { 36-channel } \\
\text { neurovascular } \\
\text { coil }\end{array}$ & NA & Yes & $\begin{array}{l}\text { T1WI (SNAP), T2*WI } \\
\text { (MERGE), TOF-MRA }\end{array}$ & $10 / 4.8$ & $250 \times 160$ & 0.8 & NA & Subj. (2) & NA† & $\begin{array}{l}\text { Vessel wall hematoma, } \\
\text { luminal stenosis or } \\
\text { occlusion, and } \\
\text { presence of infarction }\end{array}$ \\
\hline Natori et al. (9) & $\begin{array}{l}\text { Signa HDxt, } \\
\text { GE }\end{array}$ & 1.5 & 3 & $\begin{array}{l}\text { 8-channel } \\
\text { head coil }\end{array}$ & $\begin{array}{l}\text { Parallel } \\
\text { imaging } \\
\text { factor, } 2\end{array}$ & No & $\begin{array}{l}\text { T1WI (flow-sensitive } \\
\text { FSE), T2WI, } \\
\text { TOF-MRA, BPAS }\end{array}$ & $500 / 18$ & $250 \times 190$ & 0.5 & $512 \times 512$ & Subj. (1) & Kappa 0.73 & $\begin{array}{l}\text { Vessel wall } \\
\text { hematoma, intimal flap } \\
\text { or double lumen, } \\
\text { luminal stenosis, and } \\
\text { aneurysmal dilatation }\end{array}$ \\
\hline $\begin{array}{l}\text { Ogawa et al. } \\
\text { (13) }\end{array}$ & $\begin{array}{l}\text { Trillium Oval, } \\
\text { Hitachi }\end{array}$ & 3 & 3 & $\begin{array}{l}\text { 15-channel } \\
\text { head coil }\end{array}$ & NA & Yes & $\begin{array}{l}\text { T1WI, CE-T1WI (iso } \\
\text { FSE), T2WI, } \\
\text { TOF-MRA }\end{array}$ & $487 / 16$ & $170 \times 170$ & 0.9 & $224 \times 204$ & Subj. (2) & Kappa 0.90-1 & $\begin{array}{l}\text { Vessel wall hematoma, } \\
\text { intimal flap or double } \\
\text { lumen, abnormal } \\
\text { enhancement, and } \\
\text { aneurysmal dilatation }\end{array}$ \\
\hline
\end{tabular}


TABLE 3 | Continued

\begin{tabular}{|c|c|c|c|c|c|c|c|c|c|c|c|c|c|c|}
\hline \multirow[t]{2}{*}{ Source } & \multicolumn{7}{|c|}{ Protocol } & \multirow{2}{*}{ 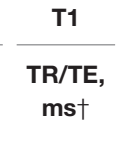 } & \multirow{2}{*}{$\frac{\mathrm{T} 1 \mathrm{WI}}{\mathrm{FOV}, \mathrm{mm}^{2} \dagger}$} & \multirow{2}{*}{$\begin{array}{c}\text { T1WI } \\
\text { Slice } \\
\text { thickness, } \\
\mathrm{mm}\end{array}$} & \multirow{2}{*}{$\frac{\text { T1WI }}{\begin{array}{c}\text { Acquisition } \\
\text { matrix } \dagger\end{array}}$} & \multirow{2}{*}{$\begin{array}{l}\text { Assessment } \\
\text { Methods } \\
\text { (reader no.) }\end{array}$} & \multirow[b]{2}{*}{$\begin{array}{l}\text { Interobserver } \\
\text { agreement } \\
\text { for diagnosis }\end{array}$} & \multirow[b]{2}{*}{$\begin{array}{l}\text { Visual (subj.) } \\
\text { assessment }\end{array}$} \\
\hline & Machine & $\mathbf{T}$ & D & Coil & $\begin{array}{l}\text { Under- } \\
\text { sampling } \dagger\end{array}$ & BB & $\begin{array}{l}\text { Sequence } \\
\text { combination }\end{array}$ & & & & & & & \\
\hline Sakurai et al. (8) & $\begin{array}{l}\text { Gyroscan } \\
\text { Intera; } \\
\text { Philips }\end{array}$ & 1.5 & 3 & $\begin{array}{l}\text { SENSE head } \\
\text { coil }\end{array}$ & $\begin{array}{l}\text { SENSE } \\
\text { factor, } 2\end{array}$ & Yes & $\begin{array}{l}\text { T1WI (VISTA and } \\
\text { 2D-BBT1WI), } \\
\text { CE-T1WI } \\
\text { (3D.-SPGR), } \\
\text { TOF-MRA }\end{array}$ & 400/13 & $180 \times 180$ & 0.9 & $256 \times 256$ & Subj. (2) & NA & $\begin{array}{l}\text { Vessel wall hematoma, } \\
\text { intimal flap or double } \\
\text { lumen, abnormal } \\
\text { enhancement, } \\
\text { aneurysmal dilatation, } \\
\text { and presence of } \\
\text { infarction }\end{array}$ \\
\hline Wu et al. (29) & $\begin{array}{l}\text { MAGNETOM } \\
\text { Verio, } \\
\text { Siemens }\end{array}$ & 3 & 3 & $\begin{array}{l}\text { 32-channel } \\
\text { head/neck } \\
\text { coil }\end{array}$ & NA & No & $\begin{array}{l}\text { T1WI (SPACE), } \\
\text { CE-T1WI (SPACE), }\end{array}$ & $900 / 14$ & $230 \times 230$ & 0.6 & $288 \times 384$ & Subj. (2) & NA $\ddagger$ & $\begin{array}{l}\text { Vessel wall hematoma, } \\
\text { intimal flap or double } \\
\text { lumen, luminal } \\
\text { stenosis, abnormal } \\
\text { enhancement, and } \\
\text { presence of infarction§ }\end{array}$ \\
\hline Yun et al. (14) & $\begin{array}{l}\text { Achieva, } \\
\text { Philips; } \\
\text { MAGNETOM } \\
\text { Skyra, } \\
\text { Siemens }\end{array}$ & 3 & 2 or 3 & $\begin{array}{l}\text { 8-channel, } \\
\text { 64-channel } \\
\text { head coil }\end{array}$ & NA & No & $\begin{array}{l}\text { T1WI, PD, CE-T1WI, } \\
\text { TOF-MRA }\end{array}$ & $\begin{array}{c}670- \\
100 / 7.6- \\
8.7\end{array}$ & $\begin{array}{c}100-170 \times \\
100-170\end{array}$ & $0.6-2.0$ & $\begin{array}{c}200-256 \times \\
200-256\end{array}$ & $\begin{array}{l}\text { Subj. + Obj. } \\
\text { (NA) }\end{array}$ & NA & $\begin{array}{l}\text { Vessel wall hematoma, } \\
\text { intimal flap or double } \\
\text { lumen, abnormal } \\
\text { enhancement, luminal } \\
\text { stenosis or occlusion, } \\
\text { aneurysmal dilatation, } \\
\text { and presence of } \\
\text { infarction }\end{array}$ \\
\hline
\end{tabular}

BB, black blood technique; BPAS, basi-parallel anatomical scanning; D, dimension; PD, proton density imaging; FSE, fast spin echo; GRAPPA, Generalized autocalibrating partially parallel acquisition (as a parallel imaging technique); MERGE, multiple echo recombined gradient echo (as a spoiled T2*-weighted sequence); MRA, magnetic resonance angiography; CE, contrast-enhanced; TOF, time-of-flight; NA, not available; SENSE, sensitivity-encoding (as a parallel imaging technique); SNAP, simultaneous non-contrast angiography and intraplaque hemorrhage (as a heavily T1WI); SPGR, spoiled gradient recalled (as a spoiled gradient echo sequence); T, Tesla; TR/TE, repetition time/echo time; (CE)T1WI, (contrast-enhanced) T1-weighted image; T2WI, T2-weighted image; VISTA, volume isotropic turbo spin echo acquisition (as a FSE). NOTE: The MRI parameters presented were that of the representative T1WI sequence; ${ }^{*} 0.66$ to 1 for wall hematoma, 0.60 to 0.89 for intimal flap or double lumen sign, 0.81 to 0.88 for aneurysmal dilatation; ${ }^{*}$ they assessed agreement between the sequences, not between reviewer; ${ }^{\ddagger} 0.91$ for intimal flap or double lumen sign and 0.90 for wall hematoma; $\$$ they evaluated only pseudoaneurysm, not outer wall dilatation. 
TABLE 4 | Pooled proportion of image findings for arterial dissection on magnetic resonance vessel wall imaging.

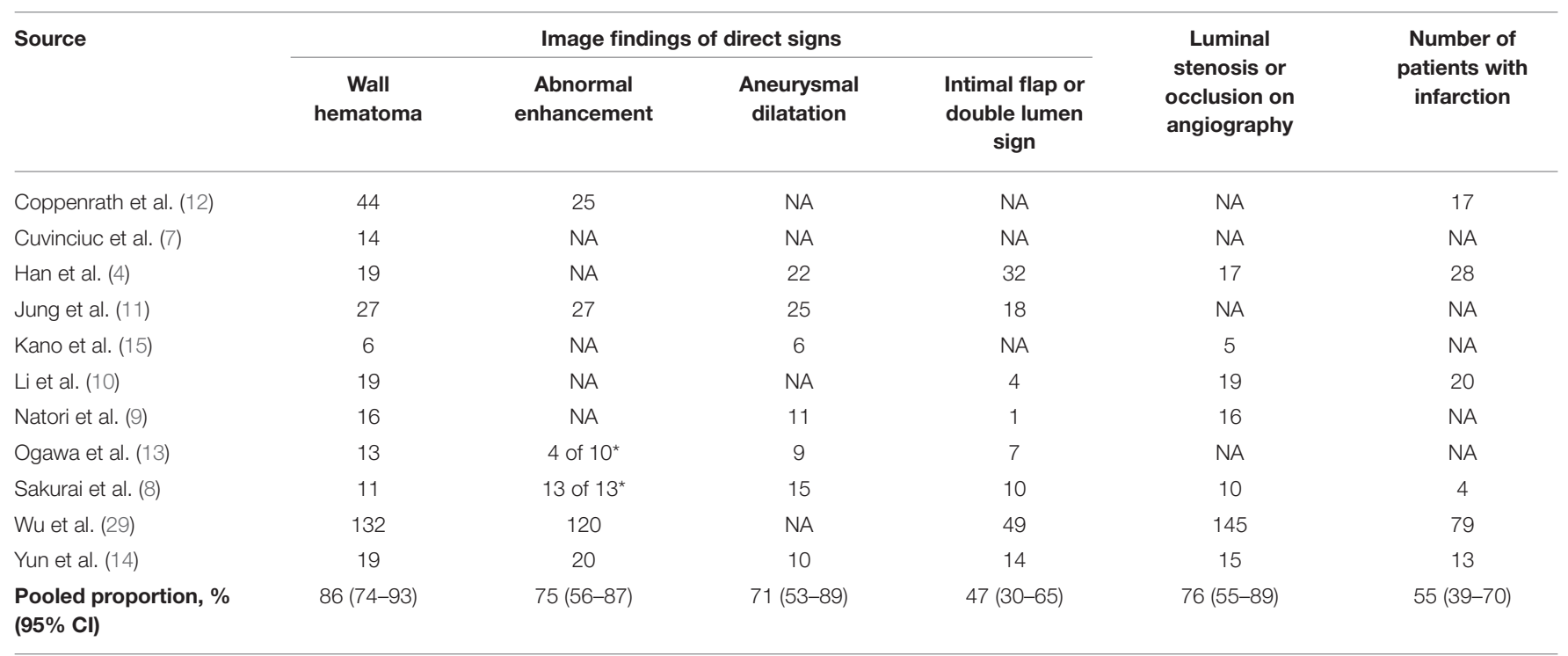

Cl, confidence interval. *All patients did not undergo contrast-enhanced imaging.

time ( $<500$ vs. $\geq 500 \mathrm{~ms}$ ), and slice thickness ( $\geq 0.6$ vs. $<0.6 \mathrm{~mm}$ ). Only the presence of CE-T1WI within the MR-VWI sequence combination was significantly associated with heterogeneity in the proportion of image findings of direct signs on MRVWI, as it affected the pooled proportion of intimal flap or double lumen signs $(p<0.001)$. All other associations were statistically insignificant.

\section{Quality Assessment}

The bias risks according to the QUADAS-2 criteria were evaluated (Figure 3). We considered low bias risk in all studies in the index test domain as they all enrolled patients based on clinico-radiologic consensus, despite the absence of surgery considering the in-nature characteristics of diagnosis of this disease. In the patient selection domain, three studies were considered to have an unclear risk of bias due to unclear blinding or non-consecutive patient enrollment $(7,13)$. In the reference standard domain, one study was considered to have an unclear risk of bias due to the absence of follow-up MRI confirmation of $\mathrm{AD}$ (12) and another due to unclear blinding to the index test (11). One study was considered to have a high risk of bias due to both unclear blinding and absence of follow-up MRI confirmation of $\mathrm{AD}$ (14). In the flow and timing domain, one study was considered to have an unclear risk of bias due to the time intervals from symptom to MRI being too heterogeneous ( 1 to 45 days) and a few suspicions of inappropriate patient exclusion (7). All studies were considered to have low applicability in the patient selection, index test, and reference standard domains.

\section{DISCUSSION}

This systematic review and meta-analysis present the pooled proportions of image findings of acute to subacute craniocervical
AD on MR-VWI. The most common findings on MR-VWI were wall hematoma, abnormal enhancement, aneurysmal dilatation, and intimal flap or double lumen signs. Among the potential covariates of heterogeneity, the presence of CE-T1WI within the MR-VWI sequence combination caused heterogeneity for the pooled proportion of intimal flap or double lumen signs. The pooled proportion of image findings provides information that can aid MR-VWI interpretation and can be used to enhance the comprehensive understanding of the radiologic diagnosis of craniocervical AD.

Recently, high-resolution MR-VWI has been widely adopted and has reported superior performance to luminal angiography in the diagnosis of $\mathrm{AD}(2,4-6)$. Also, several studies reported image findings of direct signs of dissected vessel wall on MRVWI (4, 7-15). These findings involve wall hematoma (either in the false lumen or wall layer), outer wall aneurysmal dilatation, abnormal enhancement (either in the wall or flap), and presence of intimal flap and/or double lumen signs. Wall hematoma, the most common finding in craniocervical $\mathrm{AD}$, is predominantly seen as the mural iso- to hyperintensity on T1WI (3/4). However, it can be confused as intraplaque hemorrhage in atherosclerosis, and hypointensity can also be observed on T1WI. The signal intensity on T2WI also contributes to the recognition of the stage of hematoma (2). The reason for abnormal enhancement in patients with $\mathrm{AD}$ is not fully understood yet. Inflammation, slow blood flow in the false lumen, and enhancement of the vasa vasorum are possible reasons for enhancement $(30,31)$. Aneurysmal dilatation of the outer wall can be measured at the perpendicular plane of the diseased vessel, as MRVWI allows clear discrimination between the outer wall and cerebrospinal fluid (32). Although direct visualization of the intimal flap and/or double lumen signs is the most reliable indicator, their incidence was the least in the current pooled analysis (4). 


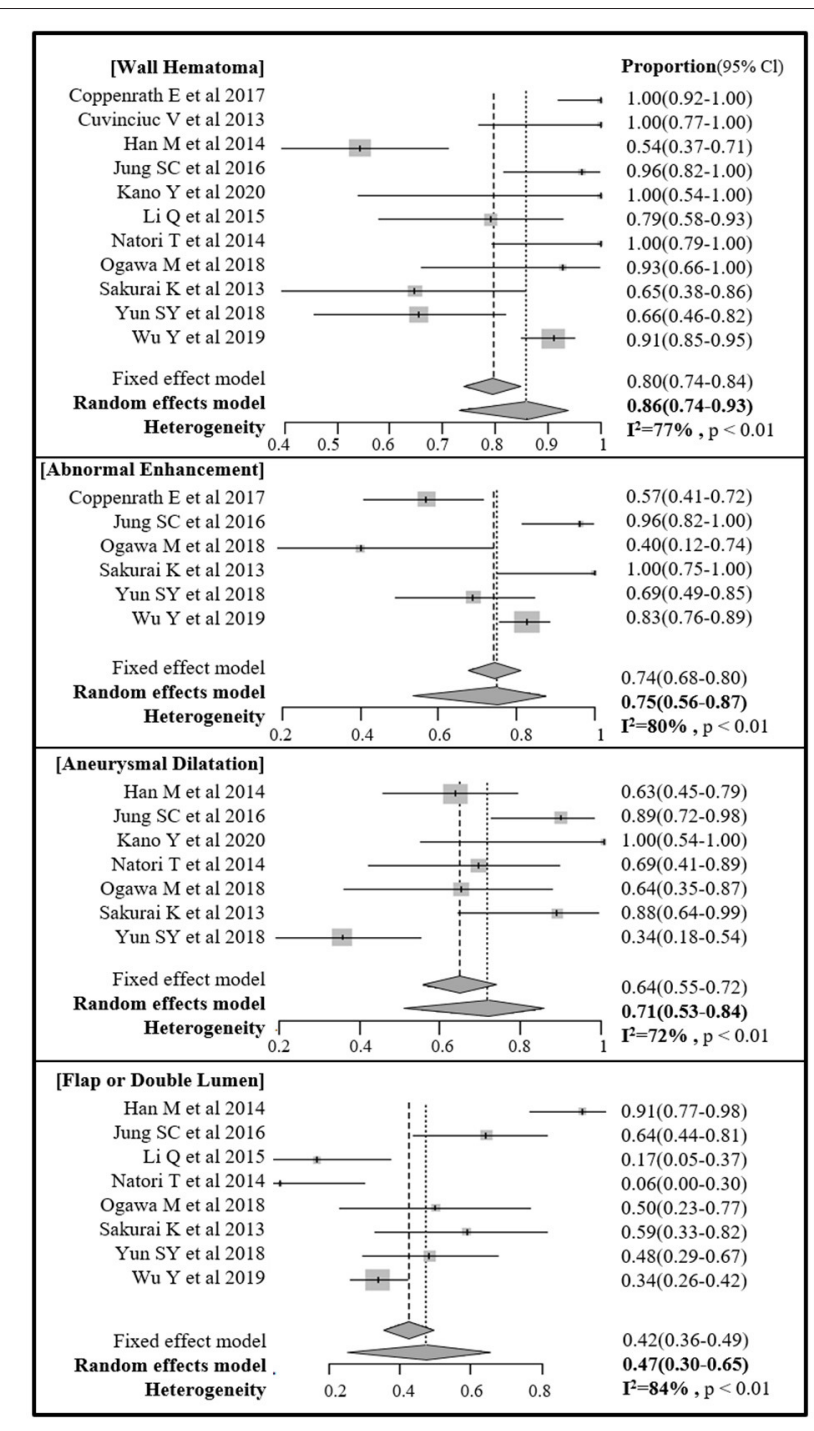

FIGURE 2 | Forest plot of pooled proportion of image findings of direct signs of craniocervical arterial dissection on magnetic resonance (MR) vessel wall imaging.

Although MR-VWI has improved the diagnostic accuracy of $\mathrm{AD}$, it remains a developing field in terms of sequence combinations, spatial resolution, and acquisition techniques (2, 4-6). MR-VWI protocols are inevitably heterologous, giving rise to the necessity for optimization. This meta-analysis also showed heterogeneity in image findings of direct signs of craniocervical $\mathrm{AD}$ across studies. In terms of sequence combinations, non-enhanced T1WI, T2WI, proton density, and enhanced T1WI sequences are generally adopted for MR-VWI (3). Han et al. (4) proposed that CE-T1WI was necessary, as they found dissection flaps in almost all cases with CE-T1WI, with substantial agreement among the readers. Correspondingly, this meta-analysis also suggests that the use of CE-T1WI within MR-VWI sequence combinations caused heterogeneity for the detection of the intimal flap or double lumen signs.
Although the effects of the other sequences could not be statistically evaluated in this meta-analysis, the combinations of non-enhanced T1WI, T2WI, proton density, and enhanced T1WI sequences are considered essential for evaluation and differential diagnosis of wall hematoma, dissection states, and vessel contours (3). The effectiveness of susceptibility-weighted imaging for detection of wall hematoma and diagnosis of craniocervical AD has also been suggested $(24,25)$. Therefore, multi-sequential combinations need to be further evaluated in terms of the protocol.

Although significance was not gauged in this meta-regression due to the relatively small number of enrolled studies, there are several factors to be considered for protocol optimization. In two of the included studies, image acquisition slice thickness was more than $1 \mathrm{~mm}(4,12)$. As the craniocervical vascular wall is a thin structure, the recommended slice thickness for MR-VWI is $<1 \mathrm{~mm}$; therefore, thicker slices need to be avoided (3). Recently, novel techniques, including the BB (32$36)$ and under-sampling techniques $(37,38)$ that improve the resolution and clinical accessibility of MR-VWI are actively being validated. Five of the studies included in this metaanalysis used the $\mathrm{BB}$ technique $(4,8,10,12,13)$; due to suppression of arterial blood and cerebrospinal fluid signals, this technique performs better in intracranial wall evaluation than 3D turbo-spin echo alone (39-42), but there are also some drawbacks, including a reduced signal-to-noise ratio of the vessel wall (32). A recent study evaluated the feasibility of the $\mathrm{BB}$ technique in the diagnosis of vertebrobasilar $\mathrm{AD}$ (5) and revealed that combining the $3 \mathrm{D}$ CE-T1WI and $\mathrm{BB}$ techniques reinforced the diagnostic performance of MR-VWI. Various under-sampling techniques, such as parallel imaging techniques, have been developed to simultaneously achieve a reduction of scan time while preserving image quality and scan range (43-45). Currently, parallel imaging techniques such as sensitivity encoding (SENSE) and generalized auto-calibrating partial parallel acquisition are limited in their ability to do this $(43,45)$ compared to non-uniform sampling techniques such as compressed sensing (CS) or combined CS-SENSE (46). Only three of the studies included in this meta-analysis used undersampling techniques, and all of these were parallel imaging techniques $(8,9,15)$. As the use of under-sampling techniques highly depends on the vendor, they are generally not easy to adopt. Thus, non-uniform sampling (CS or CS-SENSE) for craniocervical AD needs to be further evaluated.

This study had certain limitations. First, the number of included studies and their patient sample sizes were small; therefore, the statistical power of the proportion metaanalysis was low. Second, owing to the inherent scarcity of patients who undergo surgery for craniocervical $A D$, studies generally enrolled patients based on clinico-radiologic consensus. Third, as AD may present with chronologic and subsequent geometric changes after onset, we only included studies focusing on acute to subacute, and not chronic, AD. Further investigations regarding chronic $\mathrm{AD}$ and the corresponding chronologic changes are needed. Fourth, owing to the definition of MR-VWI is established recently, some papers could be missed by our searching process. Fifth, asymptomatic or mild 


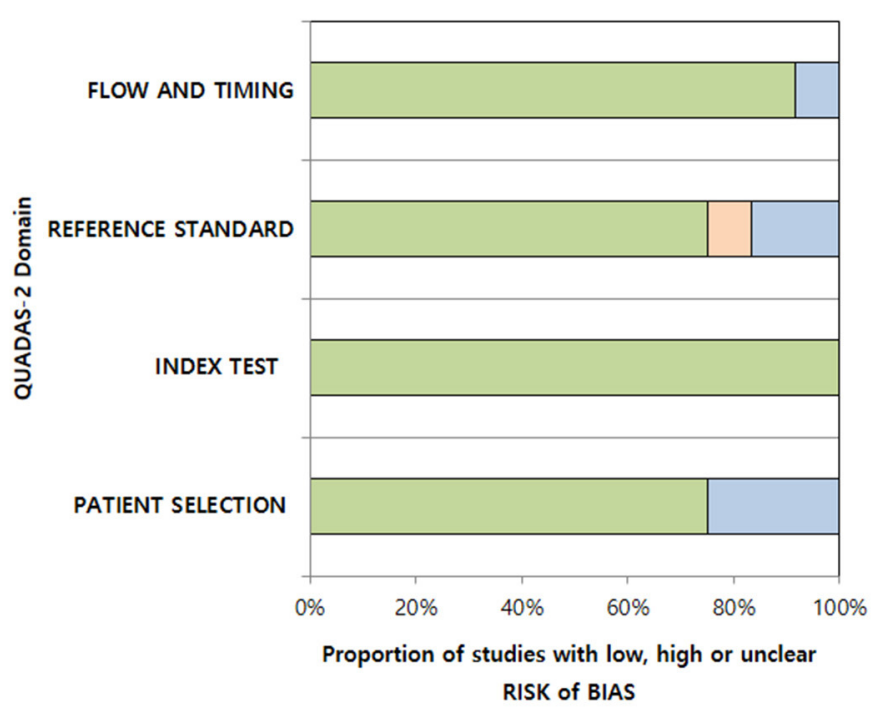

$\square$ Low $\square$ High $\square$ Unclear

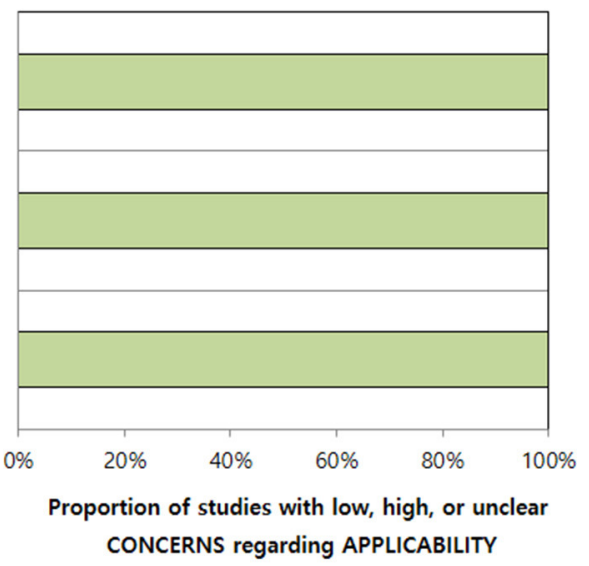

FIGURE 3 | Quality assessment of the included studies according to the Quality Assessment of Diagnostic Accuracy Studies-2 (QUADAS-2) criteria.

symptomatic patients with $\mathrm{AD}$ may be lost in the original studies due to the absence of MR-VWI in the diagnostic process. Finally, we did not perform pooled analysis for objective quantitative measurements, as only a few studies provided the required data.

\section{CONCLUSION}

Our results indicate that wall hematoma and intimal flap or double lumen signs are the most and least common direct image findings, respectively, on MR-VWI in patients with craniocervical AD. Furthermore, the absence of CE-T1WI in MR-VWI protocol was the cause of heterogeneity for the detection of the intimal flap or double lumen signs.

\section{DATA AVAILABILITY STATEMENT}

The original contributions presented in the study are included in the article/supplementary materials, further inquiries can be directed to the corresponding author/s.

\section{REFERENCES}

1. Flis CM, Jager HR, Sidhu PS. Carotid and vertebral artery dissections: clinical aspects, imaging features and endovascular treatment. Eur Radiol. (2007) 17:820-34. doi: 10.1007/s00330-006-0346-7

2. Wang Y, Lou X, Li Y, Sui B, Sun S, Li C, et al. Imaging investigation of intracranial arterial dissecting aneurysms by using $3 \mathrm{~T}$ highresolution MRI and DSA: from the interventional neuroradiologists' view. Acta Neurochir. (2014) 156:515-25. doi: 10.1007/s00701-0131989-1

3. Choi YJ, Jung SC, Lee DH. Vessel wall imaging of the intracranial and cervical carotid arteries. J Stroke. (2015) 17:238-55. doi: 10.5853/jos.2015.17. 3.238

\section{AUTHOR CONTRIBUTIONS}

SC and BC: conception and design of the study and acquisition and analysis of data. SC, BC, YB, SB, LS, and JK: drafting a significant portion of the manuscript or figures. All authors contributed to the article and approved the submitted version.

\section{FUNDING}

This work was supported by a National Research Foundation of Korea grant funded by the Korean Ministry of Science and ICT [grant number 2019R1F1A1063771] and by the Seoul National University Bundang Hospital Research Fund [grant number 092019-003]. This fund were used for grammar editing and for open access publication fees.

\section{ACKNOWLEDGMENTS}

We would like to thank Editage (www.editage.co.kr) for English language editing.

4. Han M, Rim NJ, Lee JS, Kim SY, Choi JW. Feasibility of high-resolution MR imaging for the diagnosis of intracranial vertebrobasilar artery dissection. Eur Radiol. (2014) 24:3017-24. doi: 10.1007/s00330-014-3296-5

5. Choi JW, Han M, Hong JM, Lee JS, Kim SY, Kim SS. Feasibility of improved motion-sensitized driven-equilibrium (iMSDE) prepared 3D T1-weighted imaging in the diagnosis of vertebrobasilar artery dissection. J Neuroradiol. (2018) 45:186-91. doi: 10.1016/j.neurad.2017.11.006

6. Jung SC, Kim HS, Choi CG, Kim SJ, Kwon SU, Kang DW, et al. Spontaneous and unruptured chronic intracranial artery dissection : high-resolution magnetic resonance imaging findings. Clin Neuroradiol. (2018) 28:171-81. doi: 10.1007/s00062-016-0544-x

7. Cuvinciuc V, Viallon M, Momjian-Mayor I, Sztajzel R, Pereira VM, Lovblad $\mathrm{KO}$, et al. $3 \mathrm{D}$ fat-saturated $\mathrm{T} 1$ SPACE sequence for the 
diagnosis of cervical artery dissection. Neuroradiology. (2013) 55:595-602. doi: 10.1007/s00234-013-1141-1

8. Sakurai K, Miura T, Sagisaka T, Hattori M, Matsukawa N, Mase M, et al. Evaluation of luminal and vessel wall abnormalities in subacute and other stages of intracranial vertebrobasilar artery dissections using the volume isotropic turbo-spin-echo acquisition (VISTA) sequence: a preliminary study. J Neuroradiol. (2013) 40:19-28. doi: 10.1016/j.neurad.2012.02.005

9. Natori T, Sasaki M, Miyoshi M, Ohba H, Oura MY, Narumi S, et al. Detection of vessel wall lesions in spontaneous symptomatic vertebrobasilar artery dissection using T1-weighted 3-dimensional imaging. J Stroke Cerebrovasc Dis. (2014) 23:2419-24. doi: 10.1016/j.jstrokecerebrovasdis.2014.05.019

10. Li Q, Wang J, Chen H, Gong X, Ma N, Gao K, et al. Characterization of craniocervical artery dissection by simultaneous $\mathrm{MR}$ noncontrast angiography and intraplaque hemorrhage imaging at 3T. AJNR Am J Neuroradiol. (2015) 36:1769-75. doi: 10.3174/ajnr.A4348

11. Jung SC, Kim HS, Choi CG, Kim SJ, Lee DH, Suh DC, et al. Quantitative analysis using high-resolution 3T MRI in acute intracranial artery dissection. J Neuroimaging. (2016) 26:612-7. doi: 10.1111/jon.12357

12. Coppenrath E, Lenz O, Sommer N, Lummel N, Linn J, Treitl K, et al. Clinical significance of intraluminal contrast enhancement in patients with spontaneous cervical artery dissection: a black-blood MRI study. Rofo. (2017) 189:624-31. doi: 10.1055/s-0043-104632

13. Ogawa M, Omata S, Kan H, Arai N, Asai M, Urano M, et al. Utility of the variable flip angle 3D fast-spin echo (isoFSE) sequence on $3 \mathrm{~T}$ MR for diagnosing vertebrobasilar artery dissection. Radiol Phys Technol. (2018) 11:228-34. doi: 10.1007/s12194-018-0460-7

14. Yun SY, Heo YJ, Jeong HW, Baek JW, Choo HJ, Seo JH, et al. Spontaneous intracranial vertebral artery dissection with acute ischemic stroke: highresolution magnetic resonance imaging findings. Neuroradiol J. (2018) 31:262-9. doi: 10.1177/1971400918764129

15. Kano Y, Inui S, Oguri T, Kato H, Yuasa H, Morimoto S, et al. Utility of T2weighted high-resolution vessel wall imaging for the diagnosis of isolated posterior inferior cerebellar artery dissection at acute and early subacute stages. J Neurol Sci. (2020) 411:116693. doi: 10.1016/j.jns.2020.116693

16. Liberati A, Altman DG, Tetzlaff J, Mulrow C, Gotzsche PC, Ioannidis JP, et al. The PRISMA statement for reporting systematic reviews and meta-analyses of studies that evaluate health care interventions: explanation and elaboration. Ann Intern Med. (2009) 151:W65-94. doi: 10.7326/0003-4819-151-4-200908180-00136

17. Habs M, Pfefferkorn T, Cyran CC, Grimm J, Rominger A, Hacker M, et al. Age determination of vessel wall hematoma in spontaneous cervical artery dissection: a multi-sequence 3T cardiovascular magnetic resonance study. $J$ Cardiovasc Magn Reson. (2011) 13:76. doi: 10.1186/1532-429X-13-76

18. Whiting PF, Rutjes AW, Westwood ME, Mallett S, Deeks JJ, Reitsma JB, et al. QUADAS-2: a revised tool for the quality assessment of diagnostic accuracy studies. Ann Intern Med. (2011) 155:529-536. doi: 10.7326/0003-4819-155-8-201110180-00009

19. Kim KW, Lee J, Choi SH, Huh J, Park SH. Systematic review and metaanalysis of studies evaluating diagnostic test accuracy: a practical review for clinical researchers-part I. General guidance and tips. Korean J Radiol. (2015) 16:1175-87. doi: 10.3348/kjr.2015.16.6.1175

20. Lee J, Kim KW, Choi SH, Huh J, Park SH. Systematic review and meta-analysis of studies evaluating diagnostic test accuracy: a practical review for clinical researchers-part, II. Statistical methods of meta-analysis. Korean J Radiol. (2015) 16:1188-96. doi: 10.3348/kjr.2015.16.6.1188

21. Suh $\mathrm{CH}$, Park SH. Successful publication of systematic review and metaanalysis of studies evaluating diagnostic test accuracy. Korean J Radiol. (2016) 17:5-6. doi: 10.3348/kjr.2016.17.1.5

22. Higgins JP, Thompson SG, Deeks JJ, Altman DG. Measuring inconsistency in meta-analyses. Bmj. (2003) 327:557-60. doi: 10.1136/bmj.327.7414.557

23. Kidoh M, Nakaura T, Takashima H, Yoshikawa M, Uemura S, Harada K, et al. MR diagnosis of vertebral artery dissection: value of 3D time-of-flight and true fast imaging with steady-state precession fusion imaging. Insights Imaging. (2013) 4:135-42. doi: 10.1007/s13244-012-0204-x

24. Kim TW, Choi HS, Koo J, Jung SL, Ahn KJ, Kim BS, et al. Intramural hematoma detection by susceptibility-weighted imaging in intracranial vertebral artery dissection. Cerebrovasc Dis. (2013) 36:292-8. doi: $10.1159 / 000354811$
25. Kato A, Shinohara Y, Yamashita E, Fujii S, Miyoshi F, Kuya K, et al. Usefulness of R2* maps generated by iterative decomposition of water and fat with echo asymmetry and least-squares estimation quantitation sequence for cerebral artery dissection. Neuroradiology. (2015) 57:909-15. doi: $10.1007 / \mathrm{s} 00234-015-1549-\mathrm{x}$

26. Saito A, Fujimura M, Endo H, Omodaka S, Kanoke A, Sato K, et al. Diagnostic value of contrast-enhanced magnetic resonance vessel wall imaging on the onset type of vertebral artery dissection. Cerebrovasc Dis. (2019) 48:124-31. doi: $10.1159 / 000503852$

27. Yuan $\mathrm{X}$, Cui $\mathrm{X}, \mathrm{Gu} \mathrm{H}$. Evaluating cervical artery dissections in young adults: a comparison study between high-resolution MRI and CT angiography. Int $J$ Cardiovasc Imag. (2020) 36:1113-9. doi: 10.1007/s10554-020-01799-4

28. Park KJ, Jung SC, Kim HS, Choi CG, Kim SJ, Lee DH, et al. Multi-contrast high-resolution magnetic resonance findings of spontaneous and unruptured intracranial vertebral artery dissection: qualitative and quantitative analysis according to stages. Cerebrovasc Dis. (2016) 42:23-31. doi: 10.1159/000444315

29. $\mathrm{Wu} \mathrm{Y}, \mathrm{Wu} \mathrm{F}$, Liu Y. High-resolution magnetic resonance imaging of cervicocranial artery dissection: imaging features associated with stroke. Stroke. (2019) 50:3101-7. doi: 10.1161/STROKEAHA.119.026362

30. Pfefferkorn T, Saam T, Rominger A, Habs M, Gerdes LA, Schmidt C, et al. Vessel wall inflammation in spontaneous cervical artery dissection: a prospective, observational positron emission tomography, computed tomography, and magnetic resonance imaging study. Stroke. (2011) 42:15638. doi: 10.1161/STROKEAHA. 110.599548

31. Kwak HS, Hwang SB, Chung GH, Jeong SK. High-resolution magnetic resonance imaging of symptomatic middle cerebral artery dissection. J Stroke Cerebrovasc Dis. (2014) 23:550-3. doi: 10.1016/j.jstrokecerebrovasdis.2013.03.019

32. Cho SJ, Jung SC, Suh CH, Lee JB, Kim D. High-resolution magnetic resonance imaging of intracranial vessel walls: Comparison of 3D T1-weighted turbo spin echo with or without DANTE or iMSDE. PLoS ONE. (2019) 14:e0220603. doi: 10.1371/journal.pone.0220603

33. Saam T, Habs M, Pollatos O, Cyran C, Pfefferkorn T, Dichgans M, et al. High-resolution black-blood contrast-enhanced T1 weighted images for the diagnosis and follow-up of intracranial arteritis. Br J Radiol. (2010) 83:e182184. doi: $10.1259 / \mathrm{bjr} / 74101656$

34. Pfefferkorn T, Linn J, Habs M, Opherk C, Cyran C, Ottomeyer C, et al. Black blood MRI in suspected large artery primary angiitis of the central nervous system. J Neuroimaging. (2013) 23:379-83. doi: 10.1111/j.1552-6569.2012.00743.x

35. Noorani A, Kiessewetter C, Botnar R, Figueroa CA, Henningsson M. (2015) Volumetric black-blood imaging of aortic dissection using T2 prepared inversion recovery. J Cardiovasc Magn Reson. 17:396. doi: 10.1186/1532-429X-17-S1-P396

36. Xie Y, Yang Q, Xie G, Pang J, Fan Z, Li D. Improved black-blood imaging using DANTE-SPACE for simultaneous carotid and intracranial vessel wall evaluation. Magn Reson Med. (2016) 75:2286-94. doi: 10.1002/mrm. 25785

37. Zhu C, Tian B, Chen L, Eisenmenger L, Raithel E, Forman C, et al. Accelerated whole brain intracranial vessel wall imaging using black blood fast spin echo with compressed sensing (CS-SPACE). Magma. (2018) 31:457-67. doi: 10.1007/s10334-017-0667-3

38. Jia S, Zhang L, Ren L, Qi Y, Ly J, Zhang N, et al. Joint intracranial and carotid vessel wall imaging in 5 minutes using compressed sensing accelerated DANTE-SPACE. Eur Radiol. (2020) 30:119-27. doi: 10.1007/s00330-019-06366-7

39. Wang J, Yarnykh VL, Yuan C. Enhanced image quality in black-blood MRI using the improved motion-sensitized driven-equilibrium (iMSDE) sequence. J Magn Reson Imaging. (2010) 31:1256-63. doi: 10.1002/jmri. 22149

40. Yoneyama $M$, Nakamura $M$, Takahara $T$, Takemura A, Obara $M$, Tabuchi $\mathrm{T}$, et al. Improvement of $\mathrm{T} 1$ contrast in whole-brain blackblood imaging using motion-sensitized driven-equilibrium prepared 3D turbo spin echo (3D MSDE-TSE). Magn Reson Med Sci. (2014) 13:61-5. doi: $10.2463 / \mathrm{mrms} .2013-0047$

41. Wang J, Helle M, Zhou Z, Bornert P, Hatsukami TS, Yuan C. Joint blood and cerebrospinal fluid suppression for intracranial vessel wall MRI. Magn Reson Med. (2016) 75:831-8. doi: 10.1002/mrm.25667 
42. Zhang L, Zhang N, Wu J, Liu X, Chung YC. High resolution simultaneous imaging of intracranial and extracranial arterial wall with improved cerebrospinal fluid suppression. Magn Reson Imaging. (2017) 44:65-71. doi: 10.1016/j.mri.2017.08.004

43. Petr J, Kybic J, Bock M, Muller S, Hlavac V. Parallel image reconstruction using B-spline approximation (PROBER). Magn Reson Med. (2007) 58:58291. doi: 10.1002/mrm.21366

44. Tsao J, Kozerke S. MRI temporal acceleration techniques. J Magn Reson Imaging. (2012) 36:543-60. doi: 10.1002/jmri.23640

45. Aja-Fernandez S, Vegas-Sanchez-Ferrero G, Tristan-Vega A. Noise estimation in parallel MRI: GRAPPA and SENSE. Magn Reson Imaging. (2014) 32:281-90. doi: 10.1016/j.mri.2013.12.001

46. Toledano-Massiah S, Sayadi A, de Boer R, Gelderblom J, Mahdjoub R, Gerber $\mathrm{S}$, et al. Accuracy of the compressed sensing accelerated 3D-FLAIR sequence for the detection of MS plaques at 3T. AJNR Am J Neuroradiol. (2018) 39:454-8. doi: 10.3174/ajnr.A5517

Conflict of Interest: The authors declare that the research was conducted in the absence of any commercial or financial relationships that could be construed as a potential conflict of interest.

Copyright (c) 2021 Cho, Choi, Bae, Baik, Sunwoo and Kim. This is an open-access article distributed under the terms of the Creative Commons Attribution License (CC $B Y)$. The use, distribution or reproduction in other forums is permitted, provided the original author(s) and the copyright owner(s) are credited and that the original publication in this journal is cited, in accordance with accepted academic practice. No use, distribution or reproduction is permitted which does not comply with these terms. 